Vol. 40 (1989) [357-361]

\title{
PERIODIC SOLUTIONS OF A SECOND ORDER NONLINEAR DIFFERENTIAL EQUATION
}

\author{
BaHMAN MEIRI
}

We consider the following non-linear nonautonomous second order differential equation

$$
x^{\prime \prime}(K+h(x)) x^{\prime}+f(t, x)=p(t)
$$

where $h(x)$ is continuous, $f, p$ are continuous and periodic with respect to $t$ of period $w$. Using the Leray-Schauder fixed point technique we prove that the above equation possesses at least one non-trivial periodic solution of period $\boldsymbol{w}$.

It is obvious that the linear differential equation

$$
x^{\prime \prime} k x^{\prime}=p(t), \quad p(t+w) \equiv p(t), \quad \int_{0}^{w} p(t) d t=0
$$

possesses a $w$-periodic solution. It is interesting to note that the following non-linear differential equation

$$
x^{\prime \prime}+(K+h(x)) x^{\prime}+f(t, x)=p(t)
$$

where $h$ is a continuous function, $f, p$ are continuous and periodic with respect to $t$ of period $w$, also possesses a $w$-periodic solution. The existence of periodic solutions is proved on the basis of the Leray-Schauder fixed point technique. The conditions imposed upon the non-linear terms are not very restrictive. Therefore equation (2) with those conditions has many applications.

ThEOREM 1. Differential equation (2) admits at least one $w$-periodic solution if

(i) $\int_{0}^{w} p(t) d t=0$ [that is, $P(t)=\int_{0}^{t} p(s) d s$ is $w$-periodic],

(ii) $|H(y)| \leqslant M\left[H(y)=\int_{0}^{y} h(s) d s\right]$,

(iii) $(|f(t, x)|) /(|x|) \rightarrow 0$ as $|x| \rightarrow \infty$, uniformly in $t$,

(iv) $f(t, x) \operatorname{sgn} x \geqslant 0(|x| \geqslant b)$.

Received 9 November, 1988

This work was completed while the author was on sabbatical leave from the Sharif. University of Technology, Tehran, Iran and visiting the University of California at Davis, U.S.A.

Copyright Clearance Centre, Inc. Serial-fee code: 0004-9729/89 \$A2.00+0.00. 
Proof: The proof by means of the Leray-Schauder method is simple. We consider a differential equation containing the parameter $\mu, 0 \leqslant \mu \leqslant 1$,

$$
x^{\prime \prime}+k x^{\prime}+C x=\mu\left\{p(t)-f(t, x)+C x-x^{\prime} h(x)\right\}
$$

where $C$ is an arbitrary positive constant. For $\mu=0$ we obtain a homogeneous linear equation the only $w$-periodic solution of which is the trivial one; for $\mu=1$ equation (2) is identical with the original one (1). It is a well-known fact (see $[1,2,3]$ ) that equation (3) adimits at least one periodic solution for each parameter value $\mu \in[0,1]$, if for $0<\mu<1$ all periodic solutions as well as their first derivatives are uniformly bounded. Consequently the stated theorem can be proved with the aid of an a priori estimate.

Let $x(t) \equiv x(t+w)$ be a solution of equation (3) and let $0<\mu<1$. We write

$$
R=\max _{0 \leqslant t \leqslant w}|x(t)|, \quad F=F(R)=\max _{|x| \leqslant R, 0 \leqslant t \leqslant w}|f(t, x)| .
$$

The derivative $y=x^{\prime}$ satisfies the equation

$$
y^{\prime}+k y=\mu\left\{e(t)-f(t, x(t))-h(x(t)) x^{\prime}(t)\right\}-(1-\mu) C x(t)
$$

Introducing the Green's function

$$
G(t ; s)= \begin{cases}\frac{e^{k(\Delta-t-w)}}{-1+e^{-k w}} ; & 0 \leqslant t \leqslant s \leqslant w \\ \frac{e^{k(o-t)}}{-1+e^{-k w}} ; & 0 \leqslant s \leqslant t \leqslant w\end{cases}
$$

$[G(t+0, t)-G(t-0, t)=1]$ of the boundary value problem

$$
\begin{aligned}
y^{\prime}+k y & =q(t) \\
y(0) & =y(w)
\end{aligned}
$$

where

$$
q(t)=\mu\left\{e(t)-f(t, x(t))-h(x(t)) \cdot x^{\prime}(t)\right\}-(1-\mu) \cdot e \cdot x(t)
$$

is periodic and $q(t+w)=q(t)$, we obtain the following representation of the solution of $y(t)$

$$
y(t)=\int_{0}^{w} G(t ; s) q(s) d s .
$$


Replacing $q(t)$ by the term $h(x(t)) \cdot x^{\prime}(t)$ which occurs in the expression for $q(t)$ we obtain

$$
\begin{aligned}
y(t) & =\int_{0}^{w} G(t ; s) h(x(s)) \cdot x^{\prime}(s) d s \\
& \left.=H(x(t)) G(t ; s)]_{0}^{t-0}+H(x(t)) G(t ; s)\right]_{t+0}^{w}-\int_{0}^{w} G_{t}(t, s) H(x(s)) d s \\
& =H(x(t))-\int_{0}^{w} G_{t}(t ; s) H(x(s)) d s .
\end{aligned}
$$

Inserting the explicit expression for $q(t)$ in equation (4) we derive estimates of the type

$$
|y(t)| \leqslant \rho(m+F(R)+2 M+C R)
$$

where $\rho=\max \{1,1 / k\}, m=\max _{0 \leqslant t \leqslant w}|p(t)|$.

Now a term by term integration of differential equation (2) (for the periodic solution) yields

$$
\left[x^{\prime}(t)+K x(t)+\mu H(x(t))-P(t)\right]_{0}^{w}+\int_{0}^{w}\{(1-\mu) C x(t)=\mu f(t, x(t))\} d t=0
$$

or

$$
\int_{0}^{w}\{(1-\mu) C \cdot x(t)+\mu f(t, x(t))\} d t=0 .
$$

Since $1-\mu>0$ and we have

$$
\{(1-\mu) \cdot c \cdot x(t)+\mu f(t, x(t))\} \operatorname{sgn} x=(1-\mu) C|x|+\mu f(t, x) \operatorname{sgn} x>0
$$

for $|x| \geqslant b, t \in[0, w]$, it follows that $|x(t)| \geqslant b$ for $0 \leqslant t \leqslant w$ is excluded. Therefore there exists $\tau, 0<\tau<w$, such that $|x(\tau)|<b$. Applying the mean-value thorem to an arbitrary interval $[\tau, t] \subseteq[\tau, \tau+w]$, we have

$$
\begin{aligned}
|x(t)-x(\tau)| & =|t-\tau||y(\tau+\theta(t-\tau))| \\
& \leqslant w \cdot \rho \cdot(m+F(R)+2 M I+C R),
\end{aligned}
$$

or

$$
|x(t)|<b+w \cdot \rho \cdot(m+F(R)+2 M+C R) .
$$

Hence

$$
\max _{0 \leqslant t \leqslant w} \mid x(t)=R<b+w \cdot \rho \cdot(m+F(R)+2 M+C R)
$$


Choosing $0<C<1 /(w \cdot \rho)$, we obtain

$$
1<\frac{b+w \cdot \rho \cdot(m+m)}{\ell-w \cdot \rho \cdot C} \frac{1}{R}+\frac{w \cdot \rho}{1-w \cdot \rho \cdot C} \frac{F(R)}{R} .
$$

An immediate consequence of assumption (iii) is

$$
\frac{F(R)}{R} \rightarrow 0 \text { as } R \rightarrow \infty .
$$

Therefore we conclude from (5)

$$
\begin{aligned}
R & =\max _{0 \leqslant t \leqslant w}|x(t)| \leqslant R_{0} \text { (independently of } \mu \text { ), } \\
F(R) & =\max _{|x| \leqslant R, t \in[0, w]}\left|f(t, x) \leqq F_{0}=\max _{|x| \leqslant R_{0}, t \in[0, w]}\right| f(t, w) \mid .
\end{aligned}
$$

The resulting a priori estimates

$$
|x(t)| \leqslant R_{0}, \quad\left|x^{\prime}(t)\right| \leqslant \rho \cdot\left(m+F_{0}+2 M+C R_{0}\right)
$$

ensure the existence of a periodic solution of equation (2) as we stated in our theorem. $\square$ Remark. In the case

$$
f(t, x) \operatorname{sgn} x \leqslant 0, \quad(|x| \geqslant b)
$$

we introduce a new independent variable

$$
\tau=-t
$$

and obtain a differential equation of the previous type. Thus Theorem 1 remains valid if assumption ( 1 ) is replaced by (iv)'.

As an application of our theorem consider the following differential equation

$$
x^{\prime \prime}+(1+\sin x) x^{\prime}+x^{1 / 3} \sin ^{2} t=\sin t
$$

which occurs in electric circuit theory. Obviously

$$
\begin{gathered}
p(t)=\sin t, \quad \int_{0}^{2 \pi} \sin t d t=0, \quad h(x)=\sin x, \quad H(x)=-\cos x+1 \\
|H(x)| \leqslant 2, \quad f(t, x)=x^{1 / 3} \sin ^{2} t, \quad x f(t, x) \geqslant 0 \quad(b=0),
\end{gathered}
$$

and

$$
\lim _{|x| \rightarrow \infty} \frac{|f(t, x)|}{|x|}=0
$$

that is, all assumptions of our theorem are satisfied. Hence there exists at least one $2 \pi$-periodic solution of equation (6). 


\section{REFERENCES}

[1] L. Cesari, 'Functional analysis and periodic solution of nonlinear differential equation', Contributions to Differential Equations 1 (1963), 149-187.

[2] R. Reissig, 'On the existence of periodic solutions of certain non-autonomous differential equation', Ann. Mat. Pura Appl. 85 (1970), 235-240.

[3] G. Villari, 'Contirbuti allo studio dell, esistenza di Soluzione periodiche per i Sistemi di equazioni differenziali ardinasie', Ann. Mat. Pura Appl. 69 (1965), 171-190.

Department of Mathematics,

University of California Davis,

Davis CA95616,

United States of America. 